\title{
Binary stars on the galaxy SFH
}

\author{
Zhang F. ${ }^{1,2}$, Li L. ${ }^{1,2}$, Han Z. ${ }^{1,2}$ \\ ${ }^{1}$ Yunnan Observatories, Chinese Academy of Sciences, Kunming, 650011, China \\ ${ }^{2}$ Key Laboratory for the Structure and Evolution of Celestial Objects, Chinese Academy of \\ Sciences, Kunming, 650011, China \\ email: zhangfh@ynao.ac.cn
}

\begin{abstract}
Using the Yunnan-II evolutionary population synthesis models comprising binary stars, we find that the inclusion of binary stars can raise the derived stellar metallicity $Z^{*}$ and/or age $t$ (degeneracy problem), raise the stellar mass $M^{*}$, lower the gaseous metallicity $Z_{\text {gas }}$ and star formation rate (SFR) of galaxies. This means that a few stars form recently in galaxies, while more stars form during the entire evolution process when considering binary stars. If the degeneracy between $t$ and $Z^{*}$ can be broken, its effect on the feedback process and star formation history can be determined.
\end{abstract}

Keywords. (stars:) binaries: general, galaxies: evolution.

\section{Introduction}

Evolutionary population synthesis (EPS) method is one of the most important tools to obtain the parameters, properties and evolution of galaxies. It not only can be used to derive the properties of stellar component in galaxies (for example, stellar age, metallicity, mass, etc), but also those of gasesou component (for example, gaseous metallicity) and star formation rate (SFR) in certain cases. From the co-evolution of stellar and gaseous components, we can obtain the galaxy evolution.

Binary stars are common in the Universe, especially in the massive stellar clusters (Sana 2012). So, it is necessary to consider the effect of binary stars on the galaxy evolution.

\section{EPS models and the effect of binary stars on the galaxies}

Using the Yunnan-II models, which are EPS models comprising binary interactions (BIs, Zhang et al.2004, 2005), we have obtained the effect of BIs on the determinations of stellar age $t$, metallicity $Z^{*}$, mass $M^{*}$, SFR and gaseous metallicity $Z_{\mathrm{gas}}$. The effect of BIs on the properties of stellar population (SPs) and the determinations of galaxy parameters is as follows and listed in Table 1.

- First, the inclusion of BIs makes the SPs' colors bluer, SPs' mass-to-light ratio in the K band $(M / L)_{\mathrm{K}}$ larger at all times (Table 1, the 2 nd and 3st columns). The derived stellar metallicity $Z^{*}$ and/or age $t$ (degeneracy problem) and mass $M^{*}$ of galaxies would increase. The bluer colors are caused by the fact that BIs would create some classes of hot stars, the larger $(M / L)_{\mathrm{K}}$ is because that the components in a binary system are easier to interact with each other when one is on the giant branch stage.

- Secondly, the inclusion of BIs can raise the flux shortwards of the Lyman limit $\left(F_{\lambda<912 \AA}\right)$ by $10^{2-5}$ times (Table 1, the 4 th column) for intermediate-age $\operatorname{SPs}(\log (t / \mathrm{yr})=7 \sim 8)$. The reason is that BIs can create naked helium stars $\left(T_{\text {eff }} / \mathrm{K} \sim 10^{5}\right)$. The flux $F_{\lambda<912 \AA}$ closely correlates with the gaseous component in galaxies. The inclusion of BIs makes the UV spectra harder, causing the larger $L_{\mathrm{H} \alpha}$ (the luminosity of $\mathrm{H} \alpha$ recombination line), the smaller coefficient c $\left(=\mathrm{SFR} / L_{\mathrm{H} \alpha}\right)$ and the derived SFR (Zhang et al.2012). Moreover, the radiation fields emitted by intermediateage $\left(10^{7-8} \mathrm{yr}\right)$ SPs comprising binaries are in theory possible candidates for significant central ionizing sources (CISs) of classic H II regions, while previous conclusion thought only young SPs or OB-type stars can ionize the surrounding gas. If the intermediate-age SPs are used as 
Table 1. Effect of binary interactions on the SP's properties and the determinations of galaxy parameters.

\begin{tabular}{|l|l|l|l|l|}
\hline $\begin{array}{l}\text { SPs' } \\
\text { properties }\end{array}$ & $\begin{array}{l}\text { colors } \\
\text { bluer } \\
\text { all times }\end{array}$ & $\begin{array}{l}(M / L)_{\mathrm{K}} \\
\text { larger } \\
\text { all times }\end{array}$ & $\begin{array}{l}F_{\lambda<912 \AA} \\
\operatorname{larger} \text { by } 10^{2-5} \\
\log (t / \mathrm{yr})=7 \sim 8\end{array}$ & $\begin{array}{l}F_{1000<\lambda / \AA<3000} \\
\operatorname{larger} \text { by } 10^{2} \\
\log (t / \mathrm{yr}) \sim 9.0\end{array}$ \\
\hline |reasons & $\mid$ hot stars & easier to interact & naked He stars & blue stragglers, He stars \\
\hline galaxy parameters or problem & $\mid \uparrow Z^{*}$ and/or $\uparrow t \mid \uparrow M^{*}$ & $\mid \downarrow$ SFR $\downarrow \downarrow Z_{\text {gas }}$ & $\mid$ UV-upturn problem \\
\hline information & $\begin{array}{l}\text { accumulated } \\
\text { all redshifts }\end{array}$ & $\begin{array}{l}\text { current } \\
\text { low-redshift }\end{array}$ & $\mid$ \\
\hline
\end{tabular}

the CISs of HII regions and the grid in the diagnostic diagram is used to determine the gaseous metallicity $\left(Z_{\text {gas }}\right)$, the derived $Z_{\text {gas }}$ would decrease (Zhang et al.2015).

- Thirdly, when including BIs, the flux in the range of $1000-3000 \AA\left(F_{1000<\lambda / \AA<3000}\right)$ would increase by $\sim 100$ times for old SPs $(\log (t / \mathrm{yr}) \sim 9.0)$. This is because that BIs would produce blue stragglers and helium stars. This can explain the UV-upturn problem of elliptical galaxies (Han et al.2007, Table 1, the 5th column).

\section{Implications}

In conclusion, the inclusion of BIs would raise the stellar metallicity $Z^{*}$ and/or age $t$ (degeneracy problem), raise the stellar mass $M^{*}$, lower the gaseous metallicity $Z_{\text {gas }}$ and SFR of galaxies. $Z_{\text {gas }}$ and SFR provide the information about the current activity of galaxies (longer than $10^{7-8} \mathrm{yr}$ ), $M^{*}$ embodies the accumulated information. So, it seems that galaxies are quieter at low redshift, while the accumulated stellar mass is larger when binary stars are included. If the degeneracy between $t$ and $Z^{*}$ can be broken, its effect on the feedback process and star formation history can be determined. If $t$ does not vary and $Z^{*}$ increases, it means that the galaxies are relatively active at early times because of larger $M^{*}$ and smaller SFR, the feedback efficiency is low for larger $Z^{*}$ and lower $Z_{\text {gas }}$.

Acknowledgements: This work was funded by the Chinese Natural Science Foundation (grants 11273053, 11573062, 11373063, 11390374 and 1152100017) and by Yunnan Foundation (grant 2011CI053).

\section{References}

Han Z., Podsiadlowski Ph., Lynas-Gray A. 2007, MNRAS, 380, 1098

Sana et al. 2012, nature, 444, 6

Zhang F., Han Z., Li L., Hurley J., 2004, A $\mho A, 415,117$

Zhang F., Han Z., Li L., Hurley J., 2005, MNRAS, 357,1088

Zhang F., et al., 2012, MNRAS, 421,743

Zhang F., Li, L., Han, Z., Kang, X., 2015, MNRAS, 447, L21 